\title{
Treatment of Gartlands Type II and Type III Supracondylar Fracture Humerus by Closed Reduction And Percutaneous K-wire Fixation
}

\author{
P. Sarma ${ }^{1}$, B.Agarwal ${ }^{2}$, P.Agarwal ${ }^{3}$, A.Gaikwad ${ }^{4}$ \\ Gauhati Medical College, Guwahati, Assam
}

\begin{abstract}
Introduction: Supracondylar Fracture Humerus Is One Of The Most Common Fracture Seen In Orthopaedic Clinics. Here We Report The Outcome Of Gartlands Type 2 And 3 Supracondylar Fracture In Children By Closed Reduction And Percutaneous Pinning.

Methodology: 50 Cases Of Gartlands Type 2 And 3 Supracondylar Fracture Humerus Were Treated By Closed Reduction And Percutaneous K Wire Fixation. Children From Ages 2 To 10 Were Included In The Study.

Statistical Analysis Used: Student T Test

Results:44 Patients Had Excellent Functional Outcome, 4 Patients Had Good Results, 2 Had Fair Results.

Conclusion: From Our Study We Conclude That Closed Reduction And Percutaneous Pinning Is A Safe And Efficient Method Of Fixation Of Displaced Supracondylar Fracture Of Humerus In Children.
\end{abstract}

Keywords: Supracondylar Fractures, Percutaneous Pinning, Functional Outcome.

\section{- Introduction}

Supracondylar fracture of the humerus in children is one of the most common fractures seen in orthopaedics Clinics accounting for $50 \%-70 \%$ of all elbow fracture in children in the first decade of life. The preponderance of Supracondylar fractures occurs due to various causes:

- ligamentous laxity

- $\quad$ anatomical structure of humerus - transformation from tube to flat at the lower end of humerus

Traditionally these fractures are associated with high rate of malunion, nerve palsy and vascular complications including compartment syndrome. There are two types of Supracondylar fractures of humerus in children i.e. extension type and flexion type having $97 \%$ and $3 \%$ distribution respectively. Gartland classified three types of Supracondylar fractures of humerus based on the amount of displacement at the fracture site. Type I fracture are undisplaced.Type II fractures have moderate displacement with continuity between fragments being maintained by an intact posterior cortex. Type III fractures are the most severe and have complete displacement without any contact between the fragments. Type III fractures are either posteromedial or posterolateral. Elbow fracture treatment in children remained a great challenge for surgeons since Hippocrates.Percutaneous pinning of supracondylar humerus fractures in children is an effective way to maintain an anatomic reduction after closed reduction of a displaced fracture and was first described by Casiano[4] in 1960. The goals of surgical management include correction and maintenance of alignment, avoid deformity, and early functional range of elbow movement. The aim of our study was to compare the functional results of closed reduction and percutaneous pinning in the treatment of displaced supracondylar fractures of the humerus in children..

\section{- Materials And Methods}

50 closed Supracondylar fractures (Gartlands type II \& type III) of the humerus in children were treated by closed reduction and percutaneous fixation with Kirschner wires. This study was conducted in Department of Orthopaedics, Gauhati Medical college and hospital, Guwahati.

\section{Inclusion Criteria:-}

- children of either sex of 2 to 10 years.

- children presenting within 5 days of injury

- Closed Gartlands type II \& type III Supracondylar fracture humerus, diagnosed on a plain radiograph of elbow antero-posterior and lateral view 
Exclusion Criteria:-

- Open fractures

- Supracondylar fractures with neurovascular injury which require immediate repair

- previous ipsilateral elbow fracture

- presence of any concomitant fractures in the ipsilateral limb

- Non-willing \& and unfit for surgery from anaesthesia point

All the patients selected for this study were admitted in $\mathrm{GMCH}$ and examined according to the protocol. Then AP and Lateral radiographs of effected elbow were taken. All fractures were according to Gartlands classification. After all pre operative investigations and PAC, the operative procedure was done under GA after taking Informed and written consent. Closed reduction and fixation with K-wire was done under fluoroscopy guidance. Post op radiographs were taken in both AP and Lateral views. Patients were followed up at 3 weeks, then at 4 week intervals for 3 subsequent months. POP slab were removed at 3 weeks at the time of first post op review. K-wires were removed at 3-6 weeks based on the reduction and subsequent $\mathrm{x}$ rays.

\section{- Observation And Results}

The present study consisted of 50 Supracondylar fractures of humerus ( Gartlands typeII \& type III) which were treated in the department of Orthopaedics GMCH, Guwahati.

- The patients were in the age group 2-10 years.

- Two third of the patients were boys and one third of the patients girls.

- Extension type was found to be more common then flexion type.

- Maximum no. of patients were Gartland type III.

- Left sided fractures were more common then right side

The results were classified according to FLYNN'S Criteria, as given below

\begin{tabular}{|l|l|l|}
\hline Flynn's Criteria & No. Of Cases & Percentage \\
\hline Satisfactory & & \\
\hline Excellent & 44 & 88 \\
Good & 4 & 8 \\
\hline Fair & 2 & 4 \\
\hline
\end{tabular}

In our study 44 patients had excellent result, 4 had good results and 2 had fair results

\section{- Conclusion}

From our study we conclude that closed reduction and percutaneous pin fixation is a safe and efficient method for fixation of displaced Supracondylar fractures of the humerus in children with excellent functional and cosmetic results.

\section{References}

Otsuka NY, Kasser JR: Supracondylar fractures of the humerus in children. J Am Acad Orthop Surg 1997,5:19-26.

Beaty JH, Kasser JR: Fractures about the elbow. Instr Course Lect 1995,44:199-215.

Boyd HB, Altenberg AR. Fractures about the elbow in children. Arch Surg 1944;49:213.

Casiano E: Reduction and fixation by pinning "banderillero" style fractures of the humerus at the elbow in children. Mil Med 1960, 125:262-264.

- $\quad$ Flynn JC., Mathews JG. and Benoit RL. "Blind pinning of displaced supracondylar fractures of the humerus in children". J Bone \& Joint Surg (Am),/974;56A: 263-272.

- $\quad$ Arino VL, Llurch EE, Ramriez AM, Ferrer J, Rodriguez L, Baixauli F: Percutaneous fixation of supracondylar fractures of the humerus in children. J Bone Joint Surg Am 1977, 59:914-916.

- Mostafavi HR. and Spero C. "Crossed pin fixation of displaced supracondylar humerus fractures in children". Clin Orthop, 2000; 376; 56-61.

- Ramsey RH, GRiz J. Immediate open reduction and internal fixation of severely displaced S.C. fractures of the humerus in children. Clin Orthop 1973; 90: 130

Solak, Sukru, and Aydn, Erbil: Comparision of two percutaneous pinning methods for the treatment of paediatric type III Supracondylar humerus. J.Paediatr. Orthop2003; Part B 12(5): 346-349.

- $\quad$ Skaggs DL et al.' Operative treatment of supracondylar fractures of the humerus in children'.J bone \& joint Surg, 2001;83-A(5); 735-740

- Nacht JL et al. "supracondylar fractures of the humerus in children treated by closed reduction and percutaneous pinning".Clin Orthop, 1983;177: 203-209.

- Aronson D D, and Prager BI. " Supracondylar fractures of the humerus in children- A modified technique for closed pinning". Clin Orthop, 1987; 219:174-183. 

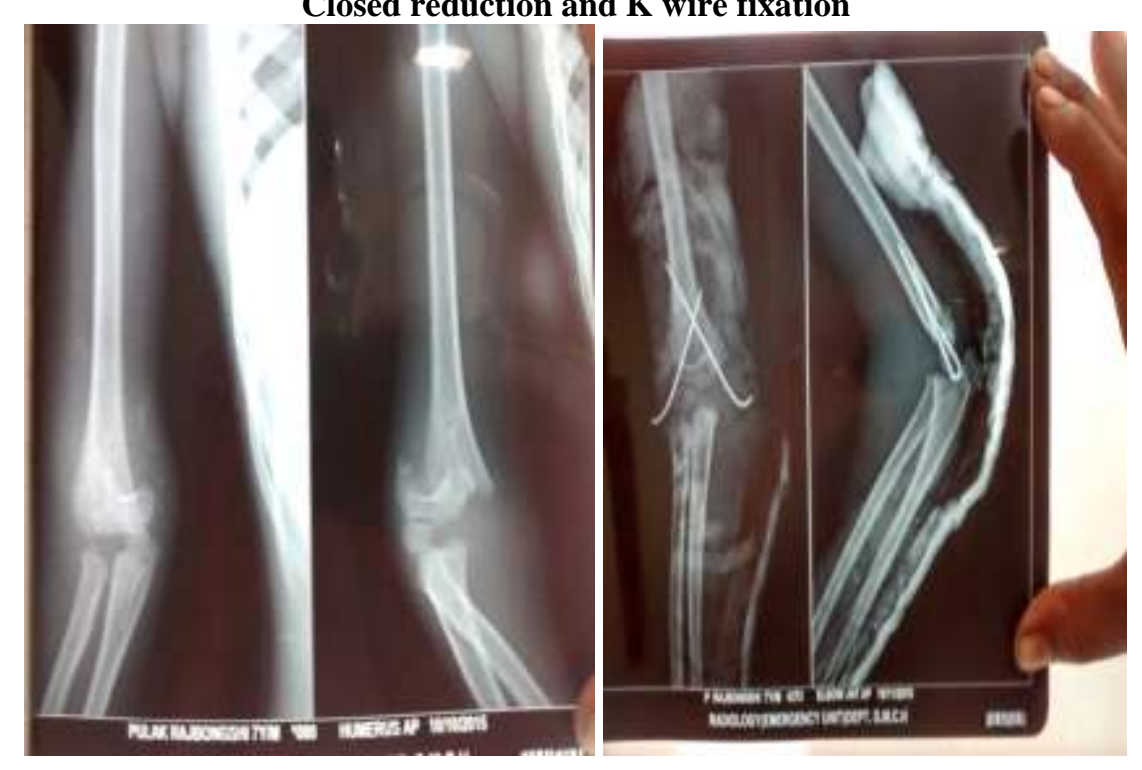

a

3 month follow-up and range of motion

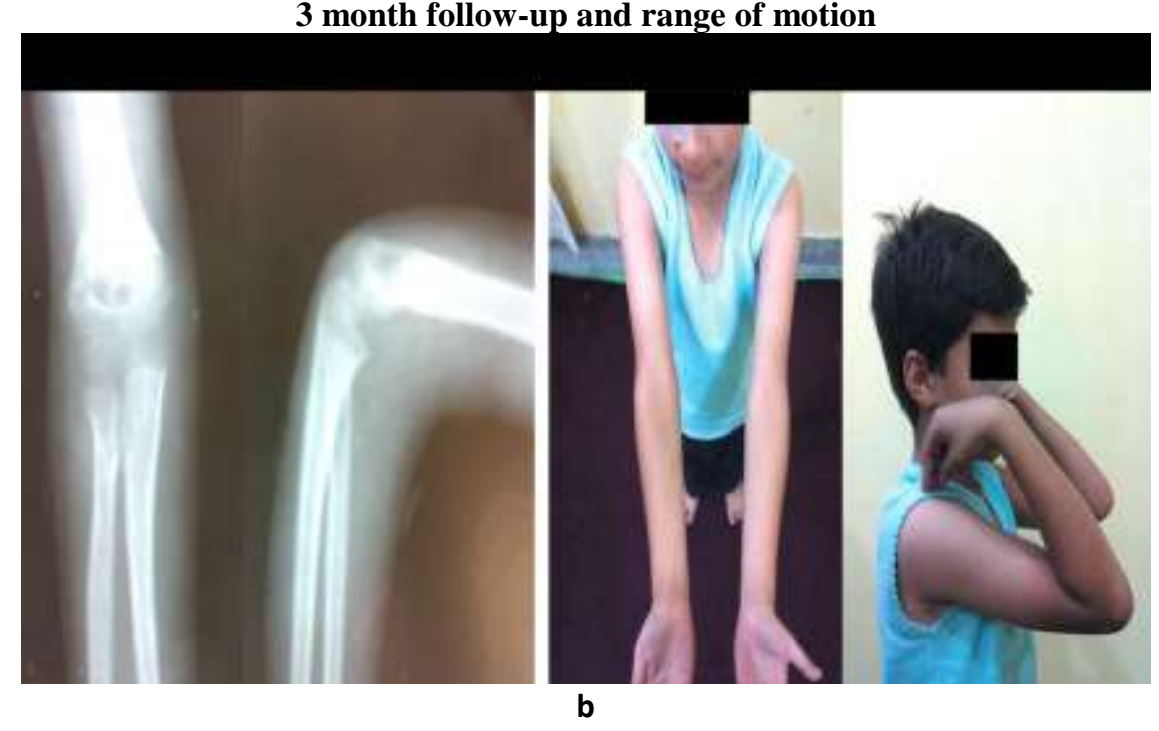

Closed reduction

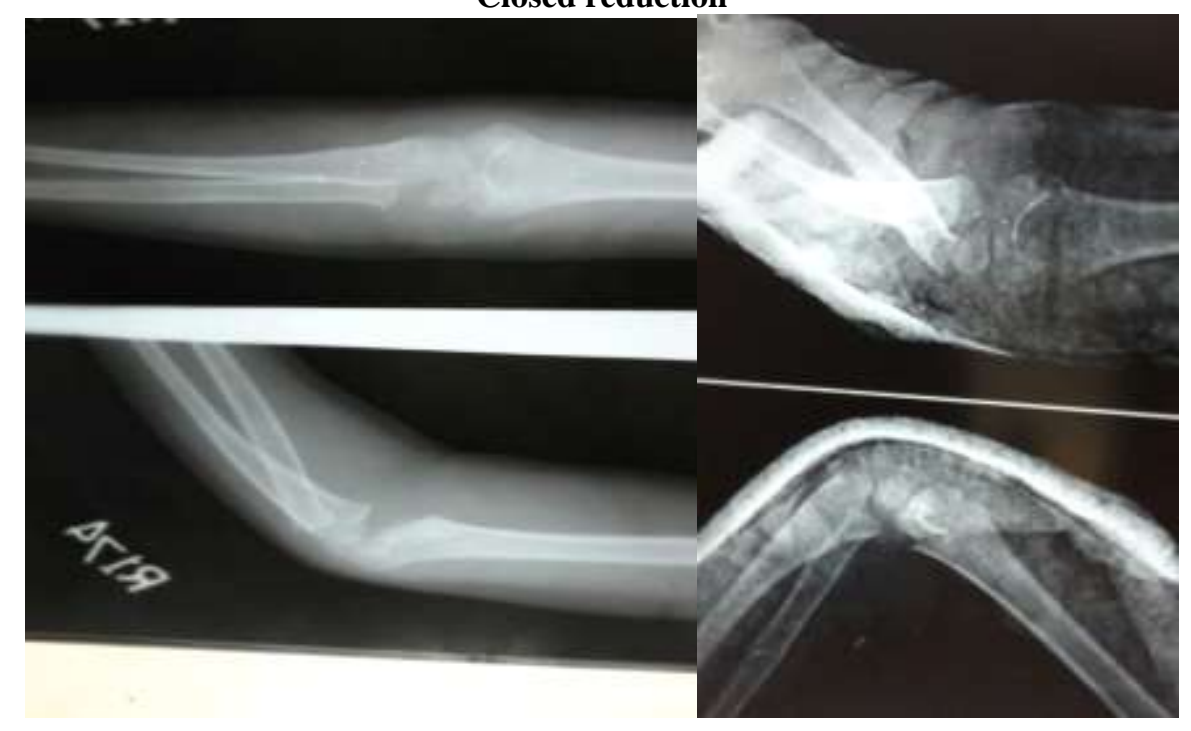


c

3 months follow-up and range of motion

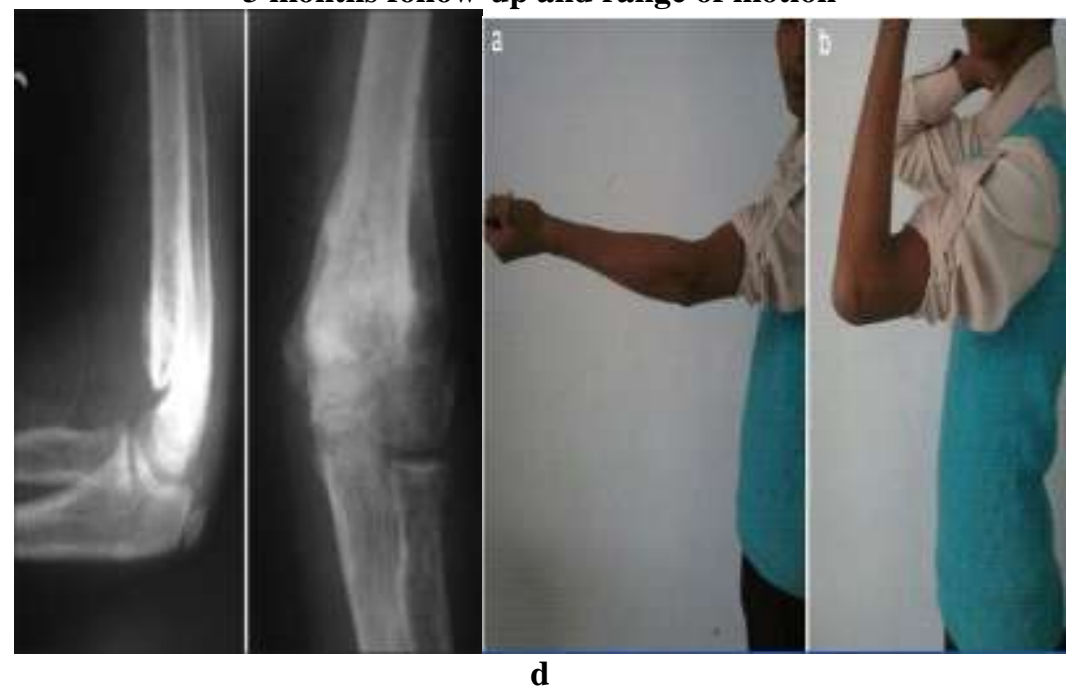

Cubitus Varus deformity

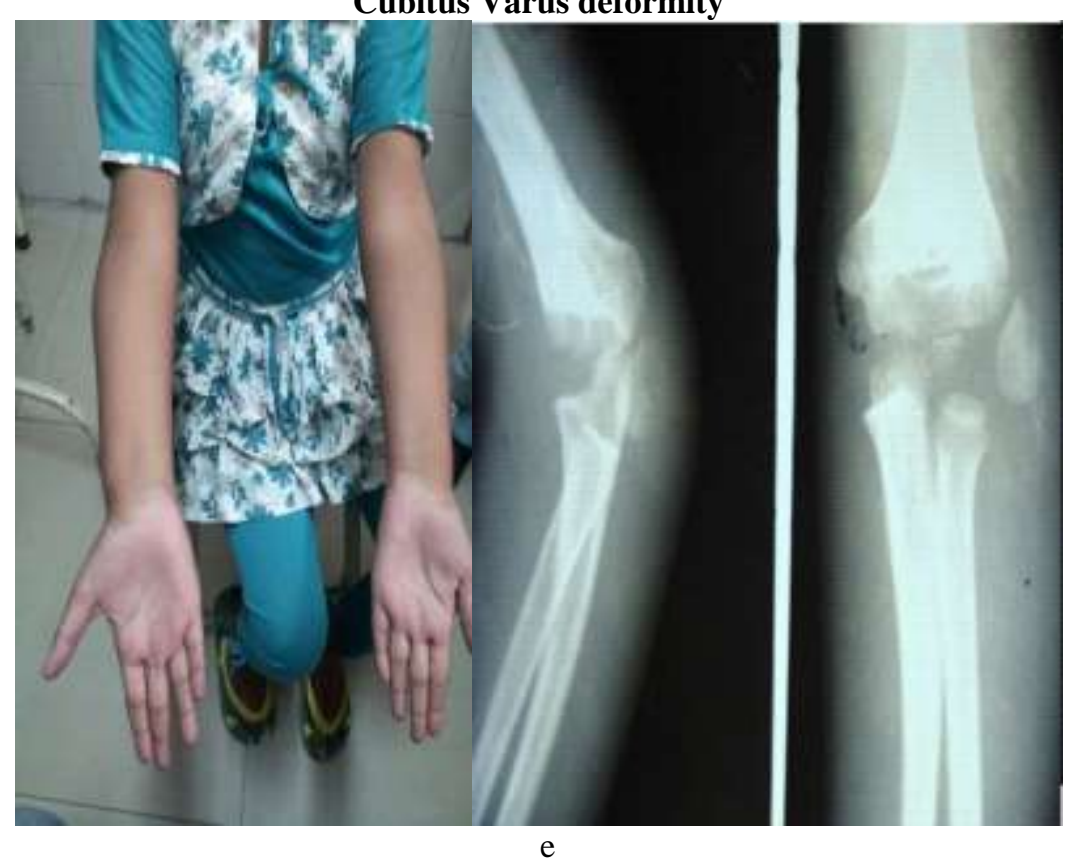

